\title{
HEIKE WEBER
}

\section{ZEIT- UND VERLUSTLOS?}

\section{Der Recycling-Kreislauf als ewiges Heilsversprechen}

\section{Einleitung}

Die circular economy ist derzeit in aller Munde, und zwar als Versprechen, das Abfallproblem ein für alle Mal lösen zu können: «Das Ende des Mülls. Wie der Kreislauf aller Stoffe gelingen kann», titelte beispielsweise im März 2020 die deutschsprachige Ausgabe von National Geographic auf dem Cover, das aufeinandergestapelte Plastikmüllsäcke zeigte. Der entsprechende Artikel erläuterte, dass der Kreis in der anvisierten Kreislaufwirtschaft geschlossen werden könne, indem alles Produzierte entweder «in <technische Nährstoffe>, aus denen neue Produkte werden», oder «in biologische Nährstoffe, die in den Boden zurückkehren», zerlegt werde. ${ }^{1}$ Das deutsche Gesetz zum Abfallumgang hieß bereits I 996 <Abfall- und Kreislaufwirtschaftsgesetz>, seit 2012 nur noch 〈Kreislaufwirtschaftsgesetz〉, als gäbe es die Abfälle nicht mehr; China hat sich 2008 der <zirkulären Ökonomie> (xunbuan jingji) verschrieben; Schottland hat 20 I 6 eine zero-waste- bzw. circular-economy-Strategie beschlossen und Frankreich vor Kurzem ein Institut National de l'Économie Circulaire auf den Weg gebracht. ${ }^{2}$

Weder die Idee einer <Kreislaufwirtschaft> jedoch ist neu noch der ihr zugrunde liegende Prozess der stofflichen Wiedernutzung von Gebrauchtem, inzwischen <Recycling〉 genannt; vielmehr haben beide das Produzieren und Konsumieren seit Jahrhunderten begleitet. Das Lumpensammeln der Frühen Neuzeit wurde mit der sogenannten Zirkulation der Stoffe assoziiert, noch ehe diese Metapher im ökonomischen und naturphilosophischen Denken weithin gängig wurde. Denn ohne Lumpen war kein Blatt Papier herzustellen - den Einsatz von Holzschliff praktizierte erst die Papierproduktion des späten i 9. Jahrhunderts. Der Druck jedes Flugblatts, jedes Briefs und jeder Zeitung bedurfte also des Einsammelns und Weiterverarbeitens von Stoffresten, um überhaupt geschrieben, verteilt oder gelesen werden zu können. Auch wenn der_die Lumpensammler_in um I900 als unhygienische, problematische
1 Robert Kunzig: Raus aus dem Müll, in: National Geographic, März 2020, 42-67, hier 65 .

$2 \mathrm{Vgl}$. Thomas Weber, Martin Stuchtey (Hg.): Deutschland auf dem Weg zur Circular Economy. Erkenntnisse aus europäischen Strategien (Vorstudie), München 2019 sowie www.circulareconomy-initiative.de (1.3.2020); Yvan Schulz, Anna Lora-Wainwright: In the Name of Circularity: Environmental Improvement and Business Slowdown in a Chinese Recycling Hub, in: Worldwide Waste. Journal of Interdisciplinary Studies, Bd. 2, Nr. 1, 2019, 1-13, doi.org/10.5334/wwwj.28. 
Sozialfigur an den Rand der Gesellschaft gedrängt worden war, blieb das Sammeln von Lumpen und Altstoffen weit darüber hinaus ein Rückgrat der Ökonomie, wie es die Berliner Zeitung Der Tag I926 in einem Artikel zu den rund 60.000 Altstoff-Sammler_innen Deutschlands zu würdigen wusste. ${ }^{3}$ «Das Leben eines Lumpen ist ein ewiger Kreislauf», so die Tageszeitung; heute noch sei er «Schiffstau auf dem Ozean», morgen an den Lumpenhandel verkauft und bald schon werde er zu Einwickelpapier.

Um auf die Zirkulation von Stoffen durch Recycling hinzuweisen oder eine solche mit dem Ziel einer Abfallvermeidung einzufordern, wurde über Jahrhunderte hinweg die Kreislauf-Figur in Anspruch genommen. Im Folgenden wird diese längere historische Tradition anhand von prägnanten Beispielen aufgezeigt. Dabei wird auch diskutiert, welche Abfall und Recycling betreffenden Herausforderungen und Problemlagen diese Metaphern vernachlässigen: Der Kreis, der ohne zeitliche Dimension - also zeitlos - ist, verspricht eine vollständige Geschlossenheit der Ströme, derweil jedes Recycling von Verlusten sowie komplexen, mehrstufigen und in Zeit und Raum verankerten Arbeits- und Transformationsprozessen gekennzeichnet ist; erst diese halten die 〈Zirkulation〉 - präziser: die Umwandlung von Resten in später wieder zu Abfall werdende Rezyklate - am Laufen. Trotz dieser augenfälligen Kontradiktion zwischen dem Ideal der Kreis(lauf)-Metapher und der Realität des Recyclings, die zunächst näher zu erläutert ist, hat das Denkmodell seit über einem halben Jahrhundert einen festen Platz in Gesellschaft, Politik und Abfallwirtschaft, wie es die folgenden Abschnitte zeigen; derweil fanden alternative Sprachbilder wie «Senke» oder «Fußabdruck» nur allmählich im Abfalldiskurs Nachhall. Erklären lässt sich dies nur historisch, nämlich mit der langen kultur- und mentalitätshistorischen Tradition des Denkens im Kreislauf sowie seiner politisch-ideellen Funktion als Heilsversprechen einer technisch-ökonomischen Lösbarkeit des Abfallproblems.

\section{Das Ideal des Kreislaufs und die Realität von «Re»cycling als «Down»cycling}

Metaphern strukturieren unser Wahrnehmen, Denken und Handeln, ${ }^{4}$ und dies meist auf subtile Weise. Selten hinterfragen der wissenschaftliche oder öffentliche Diskurs die Macht oder Eignung von eingängigen Metaphern, was ihre Deutungshoheit weiter verstärkt und Blindstellen im Verborgenen lässt. Was also macht die Wirkmacht der Denkfiguren von Kreis und Kreislauf im Bereich von Abfall und Recycling aus, wo kommen die Bilder her, was können sie zeigen und was verkennen oder verdecken sie? Kreis und Kreislauf haben im Denken über Natur und Ökonomie eine lange Tradition. Über Jahrhunderte

360.0000 Lumpensammler in Deutschland, in: Der Tag, 14.1.1926. 4 George Lakoff, Mark Johnson: Metaphors We Live By, Chicago 2003. hinweg repräsentierten sie das Ideal einer unendlichen, in sich geschlossenen Stofftransformation. Vorbild waren Selbsterhalt und Selbsterneuerung der Natur in ihrem zyklischen Werden und Vergehen - ein Denkmodell, das bis 
heute wirkt, etwa wenn wir von <natürlichen> oder <biogeochemischen〉 Kreisläufen sprechen, um den Wasserhaushalt der Erde, den Stickstoffmetabolismus des Ökosystems oder die sich über Jahrmillionen vollziehenden geologischen Vorgänge in Erdmantel und Erdkruste zu beschreiben. Über lange Zeit als von Iteration und Stetigkeit geprägt und damit der Zeit enthoben wahrgenommen, sind einige dieser fundamentalen Stoffkreisläufe von der Menschheit allerdings inzwischen maßgeblich überformt worden.

Der Uroboros - die den eigenen Schwanz verschlingende Schlange (Abb. I) - stand über Jahrhunderte für das unendliche Werden und Vergehen der Natur. Das Bildmotiv findet sich in archaischen Kulturen ebenso wie in der alchemistischen Lehre. Es symbolisierte die kosmischen und natürlichen Kreisläufe, deren Unendlichkeit und ewige Wiederkehr sowie die göttliche Eigenschaft der Natur, sich zugleich zu verbrauchen und zu erneuern. Im Uroboros gehen Anfang und Ende ineinander über; die Figur bleibt zeitlos und gibt in ihrer Anordnung keine Richtung vor. Auch in anderen bildlichen Repräsentationen - etwa zum Kosmos und Planetensystem - steht der Kreis, der weder Anfang noch Ende kennt, vornehmlich für Perfektion, Einheit und Unendlichkeit, außerdem für Einfachheit; in Natur-Bildern wie den Jahreszeiten oder dem Lebensrad symbolisiert er darüber hinaus Iteration und Zyklizität in einer als ewig gedachten Zeit. ${ }^{\mathbf{5}}$

Zeitlichkeit und Zeitvergehen lassen sich im Bild aufgrund der Gleichzeitigkeit des Gezeigten nur indirekt andeuten, etwa durch Pfeile oder räumlich geordnete Abfolgen; im Falle der Kreis-Figur ließe sich beispielsweise das Ziffernblatt einer Uhr nennen, das zugleich Iteration repräsentiert. In historischen Diagrammen ist daher die Linie, nicht der Kreis das visuelle Grundelement; die kreisrunde, papierne Volvelle (discus chronologicus), die Christoph Weigel in den I $720 e r$ Jahren als Übersicht über alle Herrscher verschiedener Regionen seit dem I. Jahrhundert n. Chr. schuf, zeigt das epistemische Problem unmittelbar auf: Dort, wo das Maul des Uroboros seinen Schwanz aufnimmt, treffen die chronologisch fernsten Figuren - so etwa Augustus und Karl VI. - räumlich aufeinander. ${ }^{6}$

Das englische cycle bezeichnet den Kreis ebenso wie den Kreislauf oder als Verb auch das Radfahren. Der Kreislauf jedoch und das Rad benötigen ein Movens, das sie am Laufen hält. Es ist der - dem Uhrzeigersinn folgende - Pfeil, der in den reduktionistischen Recycling-Piktogrammen das aktive Zirkulieren andeutet, damit aber auch trügerischerweise eine immerwährende Zyklizität und Geschlossenheit suggeriert. Die Stoffe könnten, so lautet das Versprechen, verlustlos und quasi von selbst, ähnlich dem Selbsterhalt der Natur, immerfort «kreisen» - ein Heilsversprechen, das jedem Wissen um Entropie und die Irreversibilität der Zeit ebenso widerspricht wie den technischökonomischen Realitäten von Recycling und der durch den Massenkonsum ausgelösten Dissipation von Stoffen.

Erstens ist jedes Recycling materialökonomisch betrachtet ein Downcycling: Es ist unweigerlich mit Stoff- oder Qualitätsverlusten oder auch der Entstehung
5 Jessica Helfand: Reinuenting the Wheel, New York 2002; Manual Lima: The Book of Circles. Visualizing Spheres of Knowledge, New York 2017, v. a. 32-41.

6 Daniel Rosenberg, Anthony Grafton: Cartographies of Time: A History of the Timeline, New York 2010; zum discus chronologicus vgl. 103, 105. 
neuer Reste verbunden. Materialqualitäten sinken im Laufe des Recycling-Prozesses, der daher bei jedem Material irgendwann ein Ende findet. Ohnehin geht Recycling materialspezifisch vor: Schrott beispielsweise ist leichter zu rezyklieren als Plastik und die Sortenreinheit der Abfälle entscheidet über die Qualität der Rezyklate. Manche der prozessierten Stoffe, etwa Schlämme des AltpapierRecyclings oder toxische Chemikalien beim Elektronikschrott, sollten ob der von ihnen ausgehenden Umweltgefährdung zudem nicht im Kreislauf gehalten werden. Recycling operiert mithin fernab der Totalität und Einheitlichkeit des Uroboros-Gedankens.

Zweitens erfordert die mit dem Kreis(lauf) symbolisierte Stofftransformation komplexe, zeitlich und räumlich aufeinander abgestimmte technisch-ökonomische Prozesse: Es ist aufwändige, oft unangenehme und teils gesundheitsgefährdende Müll-, Trenn- und Transportarbeit zu leisten, die beim Zusammensammeln der nach der Warendistribution und -konsumtion weit verstreuten Abfälle beginnt und beim Rezyklat endet. Der <Kreislauf〉 der angeblich <geschlossenen〉Stoffströme muss also von Müllarbeiter_innen, Technik und Maschinen kontinuierlich am Laufen gehalten werden. Der Distributionslogistik der Stoff- und Warenverteilung - von der Erzmine bis zum Supermarkt - steht als Pendant eine umfassende reversible Logistik gegenüber, die in unserem Denken, Reden und Handeln zum Müll bisher weitgehend im Unsichtbaren verbleibt. Wäre es daher nicht an der Zeit, unsere Sprachbilder kritisch zu hinterfragen, statt den etablierten Denkmustern $\mathrm{zu}$ folgen?

\section{Denktraditionen: Der Kreislauf im Abfalldiskurs um 1900}

Mit dem lateinischen circulatio oder auch dem französischen bzw. englischen circulation verwiesen Alchimisten, Physiologen oder auch ökonomische Denker der Frühen Neuzeit auf Transmutationen und Bewegungen von Stoffen, Körpersubstanzen oder Waren oder auch von Luft, Geld oder Reichtum in Natur, Körper und Gesellschaft. Als sich im Deutschen das Lehnwort der Zirkulation im I9. Jahrhundert eingebürgert hatte, wurde es auch zu einem

7 Engelbert Schramm: Im Namen des Kreislaufes. Ideengeschichte der Modelle vom ökologischen Kreislauf, Darmstadt 1997; Andreas B. Kilcher: Assimilation und Zirkulation. Ein universalistisches Wissensmodell des 19. Jahrhunderts, in: Nach Feierabend. Zürcher Jahrbuch für Wissensgeschichte, Nr. 7: Zirkulationen, 2011, 15-36.

8 Vgl. als Übersicht Heike Weber: Material Flows and Circular Thinking, in: Sebastian Haumann, Martin Knoll, Detlev Mares (Hg.): Concepts of Urban-Enuironmental History, Bielefeld 2020, 125-143. bestimmenden Schlagwort des nun aufkommenden Abfalldiskurses: ${ }^{7}$ Die zunehmenden Reste von Produktion und Konsumtion wurden in den wachsenden Städten zur Hygienegefahr, da sie sich nur noch schwerlich dem gesellschaftlichen Metabolismus wiedereingliedern ließen. Im Bereich von Stadthygiene und -sanierung wurden Zirkulation und Kreislauf zu Leitbildern, anhand derer bestehende und geplante Entsorgungssysteme kritisiert, protegiert oder neu etabliert wurden.

Für Fäkalien und Abwässer ist dies bereits untersucht. ${ }^{8}$ Um diese Reste aus den bewohnten Gebieten ausleiten zu können, legten Städte Kanalisationsinfrastrukturen an, die keinesfalls unumstritten waren: Mit dem Bild eines aufrechtzuerhaltenden $<$ Kreislaufs $>$ argumentierten einige Stadtpolitiker oder auch 
Chemiker für Alternativen wie z. B. die Anlage von Rieselfeldern vor den Toren der Stadt; diese nutzten die Abwässer als Dung, statt die Nährstoffe der Fäkalien ungenutzt wegzuschwemmen. Noch I 894 rechnete der Stadthygieniker Theodor Weyl für Berlin nach, welches Ausmaß an Nährstoffaustrag das Berliner «Kanalwasser» bewerkstellige und wie viel des Austrags auf den Rieselfeldern lande; seine Gesamtbilanz der von der Berliner Bevölkerung über Nahrungsmittel aufgenommenen und durch Fäkalien ausgeschiedenen Nährstoffe war ein Plädoyer dafür, die mit der Kanalisation einhergehenden dramatischen Verluste möglichst durch Rieselfelder zu verringern. ${ }^{9}$

Ein solches Kreislauf-Denken spielte gleichermaßen bei einem wenig später aufkommenden Entsorgungsproblem eine Rolle: demjenigen der städtischen Abfälle. Tatsächlich bildeten sich die Begriffe von <Abfall> und <Müll> in ihrer heutigen Bedeutung auch erst im späten I9. Jahrhundert entlang dieser neuen Problematik heraus. ${ }^{10}$ Zuvor waren Reste stoff- und herkunftsspezifisch klassifiziert und bezeichnet worden und vieles davon - etwa Exkremente, Schrott oder Lumpen - wurde wiederverwendet, anderes in Gruben oder auf Haufen geworfen und der Vergänglichkeit preisgegeben. Erst die Akkumulation von Resten in der wachsenden Stadt und sich ändernde technisch-ökonomische Verhältnisse brachte «das Müll» als Herausforderung der Stadt auf, und es wurden um I 900 mehr und mehr kommunale Müllabfuhren etabliert, um die Abfälle aus der Stadt zu transportieren. Meist wurden sie dann an der Peripherie auf Halden abgelagert, teils aber auch nach einer gewissen Verwesungszeit als «Müllerde» agrarisch genutzt.

Damit endete das Reste-Wiederverwenden aber keinesfalls abrupt zugunsten des Wegwerfens. Im Paris des I 9. Jahrhunderts wurden so z. B. neben Lumpen auch Knochen und die Fäkalien der Haushalte systematisch gesammelt und rezykliert. ${ }^{11}$ In den Großstädten zog eine Schar von Lumpensammler_innen von Haus zu Haus, um Reste aufzukaufen und damit Geld im Altstoffgewerbe zu verdienen; die Ärmsten der Armen suchten den Müllstrom auf seinem Weg von der kommunalen Mülltonne zur Ablagerung auf Brauchbares ab. ${ }^{22}$ Darüber, ob weggeworfen oder wiederverwertet wurde, bestimmten die jeweiligen Stoffökonomien, Produktionsweisen und Kostenrelationen zwischen Rohstoffen, Energie und Arbeitskraft. Oder auch der Wille der jeweiligen Kommune, Geld in die Müllentsorgung zu investieren: So finanzierte beispielsweise Charlottenburg mit der sogenannten Dreiteilung eine Müllabfuhr, bei der (I) Küchenund Speisereste, (2) Aschen der Kohleöfen und (3) Sperrstoffe wie Papier, Porzellan oder Schrott getrennt abgeholt und so weit als möglich weiterverwertet wurden. Der Sachbuchautor Hans Dominik sah darin einen «Rückmarsch in die Industrie»: Porzellan und Steingut wurden zermahlen, um sie als feuerfeste Materialien abzusetzen; das aus Metallgegenständen zurückgewonnene Zinn verwendete die Textilindustrie zur Beschwerung von Seidenstoffen. ${ }^{13}$ Zwar lohne nicht jede Stofftransformation, etwa das Abkratzen von Emaille von Geschirr, aber sonst läge der Rest auf ewig irgendwo nutzlos umher. Bei der
9 Vgl. Theodor Weyl: Versuch über den Stoffwechsel Berlins. Eine hygienisch-statistische Untersuchung, Berlin 1894.

10 Ludolf Kuchenbuch: Abfall. Eine Stichwortgeschichte, in: Soziale Welt, Sonderband 6: Kultur und Alltag, hg. von Hans-Georg Soeffner, Göttingen 1988, 155-170.

11 Sabine Barles: L'inuention des déchets urbains. France 1790-1970, Seyssel 2005.

12 Heike Weber: Den Stoffkreislauf am Laufen halten. Restearbeit und Resteökonomien des 20. Jahrhunderts, in: Kijan Espahangizi, Barbara Orland (Hg.): Stoffe in Bewegung. Beiträge zu einer Wissensgeschichte der materiellen Welt, Zürich 2014, 145-171.

13 Hans Dominik: Verwertung des Großstadtmülles, in: Über Land und Meer, Nr. 43, 1914, $1114 \mathrm{f}$. 
14 Vgl. Anton Lübke: Das Gold in der Mülltonne, in: Zeitungsausschnittsammlung der Sammlung Erhard, Umweltbundesamt Dessau, 1938 (B S. VI, 17); ders: Das deutsche Rohstoffwunder. Wandlungen der deutschen Rohstoffwirtschaft, Stuttgart 1938.

15 Tobler: Müllaussortierung oder Haushaltssammlung, in: Die Städtereinigung, 1937, 319-320, hier 320.

16 Heike Weber: Nazi German Waste Recovery and the Vision of a Circular Economy. The Case of Waste Paper and Rags, in: Business History, 2021 (im Erscheinen).

17 Vgl. Ruth Oldenziel, Heike Weber: Introduction. Reconsidering Recycling, in: Contemporary European History, Bd. 22, Nr. 3: Social History of Recycling and Re-use in the Twentieth Century, 2013, 347-370; Christian Möller: Der Traum vom ewigen Kreislauf. Abprodukte, Sekundärrohstoffe und Stoffkreisläufe im «Abfall-Regime» der DDR (1945-1990), in: Technikgeschichte, Bd. 81, Nr. 1, 2014, 61-89.

18 Eine ökologische Revolution behauptet z. B. Joachim Radkau: Die Ära der Ökologie. Eine Weltgeschichte, München 2011.

19 Naturkautschuk wurde seit dem späten 19. Jahrhundert großindustriell verwendet; es entstanden unmittelbar Märkte für Alt- und Regeneratgummi, vgl. Friedrich Gräbner: Gummirecycling. Altgummi: Abfallproblem oder Wertstoff?, in: Ulrich Giersch, Ulrich Kubisch (Hg.): Gummi - die elastische Faszination, Berlin 1995, 344-347.

20 Auch das Piktogramm für «verantwortungsvolle Konsumund Produktionsmuster» der 17 UN-Nachhaltigkeitsziele greift auf die Abstraktion des Möbiusbandes zurück; Grundform ist die liegende Acht, das Unendlichkeitszeichen der Mathematik. «ganzen Müllproduktion», so Dominik, handele es sich nämlich «um den Teil eines natürlichen Kreislaufes» und es sei «niemals gut», einen «solchen Kreislauf willkürlich» zu unterbrechen.

Die Kreislauf-Metapher begleitete die Geschichte des Mülls also von Anfang an und bald trat ein weiterer Begriff hinzu: das $\langle$ Recycling $>$ als Wortverbindung von <etwas rückgängig machen〉 (re) und 〈Kreis〉 (cycle). In den I920er Jahren verwies die amerikanische Petrochemie mit dem neuen Begriff auf das Zurückleiten von Residuen der Öl-Herstellung in den Raffinationsprozess; breite Verwendung fand er allerdings erst knapp vier Jahrzehnte später. Davor waren es insbesondere das NS-Regime sowie die DDR, welche ihre Abfallpolitik auf die Kreislauf-Metapher zu stützen suchten. Seit I935 etablierte das NS-Regime mehr und mehr spezifische Programme des Reste-Sammelns und -Verwertens und die NS-Propaganda instrumentalisierte bekannte Topoi wie die Rede vom «Gold in der Mülltonne» für die von ihr geforderte «restlose» Müllverwertung. ${ }^{14}$ Als Ziel formulierte ein Repräsentant der NS-Abfallpolitik I937, «alle verwertbaren Altstoffe zu erfassen und dem Rohstoffkreislauf wieder zuzuführen», ${ }^{15}$ und zwischen 1940 und 1944 wurde die Bevölkerung über das Sprachbild des Kreislaufs dazu mobilisiert, auch den letzten Rest zu sammeln und für die kriegerischen Zwecke des Regimes herzugeben. ${ }^{16}$ In der DDR wiederum wurde ab den I96oer Jahren an einer explizit am <Kreislauf $>$ orientierten Materialökonomie gearbeitet, um angesichts der Knappheit von Ressourcen Abfälle stofflich auszubeuten. ${ }^{17}$

\section{Unendliche Bänder und Pfeile im Kreis: Piktogramme eines 〈grünen> Recyclings}

Erst die Umweltpolitik der I97oer Jahre machte aus der teils nationalökonomisch, teils materialökonomisch motivierten Strategie des stofflichen Wiederverwertens eine <grüne> Praktik eines angeblich umweltbewussten $<$ ökologischen> Zeitalters. ${ }^{18}$ Seit den I97oer Jahren wurde das Recycling von Altglas, Altpapier und organischen Abfällen in der Umweltpolitik und im Umweltbewusstsein der Bürger_innen westlicher Industrienationen als ökologisch begriffen und durch politischen Druck schließlich auch auf weitere Abfälle ausgedehnt.

Bereits I 970 veröffentlichte die soeben als nationale Umweltschutzbehörde gegründete amerikanische Environmental Protection Agency eine erste Übersicht zu Recycling-Märkten und reagierte damit auf den Resource Recovery Act. Vermutlich auch, weil zeitgenössische plakative Bilder des Kreises noch fehlten, griff das Titelbild der Studie auf den Uroboros (Abb. I) zurück. Im Text wird die Übernahme der Zeichnung aus Abraham Eleazars Uraltes chymisches Werk ( 1760 ) explizit vermerkt und festgestellt, das Symbol habe «a modern counterpart in the concepts of recycling and ecology which are frequently represented by a modern circular symbol». Die Schwerpunkte 
der Studie lagen auf den quantitativ wichtigsten Märkten, und zwar von Papier, Metallen, Glas, Textilien sowie Gummi, Plastik, Schlachthofabfällen und Bauschutt. Weitgehend waren dies also jene Bereiche, in denen schon seit Jahrhunderten rezykliert wurde, erweitert um Gummi ${ }^{19}$ sowie Plastik, dessen globale Volumenproduktion diejenige des Stahls bald übertreffen sollte, das aber ob seiner stofflichen Heterogenität ganz im Gegensatz zum Schrott-Stahl-Kreislauf nur unter hohem Aufwand und mit starken Qualitätsverlusten zu rezyklieren ist. Für jede Industrie wurden Daten zum Rohstoffverbrauch und dem, was später Recycling-Quote genannt werden sollte, aufgeführt, wobei diese Quote selbstredend stets weit unter den roo Prozent eines geschlossenen Kreislaufs lag. Der Uroboros war hier Versuch, zu einem Mehr an Recyling aufzufordern.

Nicht der Uroboros, sondern seine technizistische, reduktionistische Form eines mit einem Pfeil versehenen Möbiusbandes oder Kreises hat sich in der Folgezeit als Ikone des <grünen> Recyclings durchgesetzt. Abbildung 2 zeigt das inzwischen weithin bekannte Recycling-Logo von Gary Anderson. Der damalige Architekturstudent hatte es 1970 bei einem Wettbewerb eingereicht, den ein Verpackungspapier-Hersteller (Container Corporation of America) anläss-
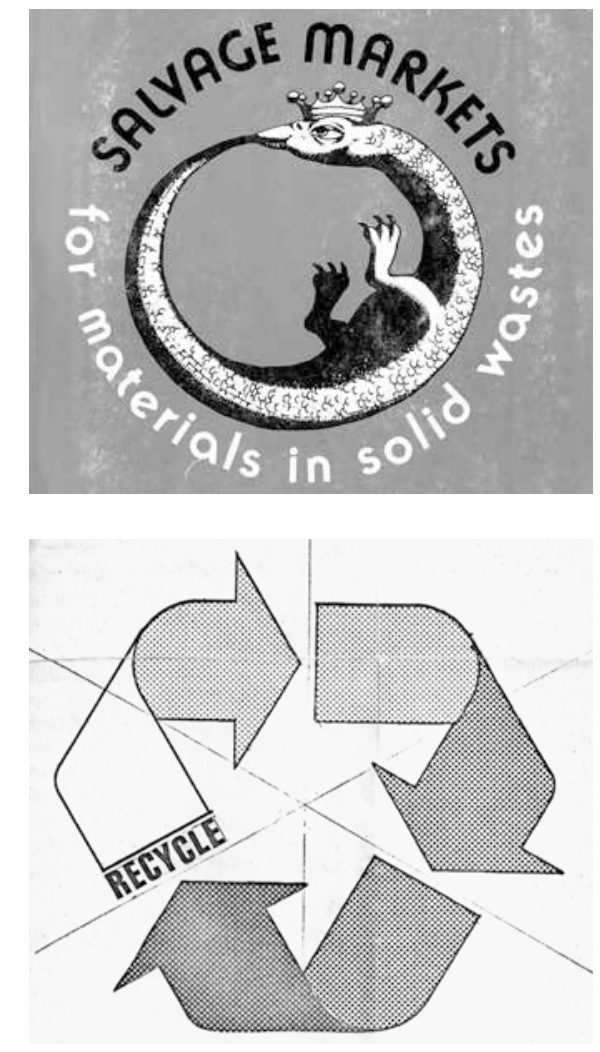
lich des Earth Day initiiert hatte. Wo sich einst der Schwanz der Schlange in deren Maul wiederfand, leiten nun drei Pfeile in Form eines Möbiusbands ineinander über, um eine unendliche, geschlossene Zirkulation zu symbolisieren. ${ }^{20}$

Mit dem Recycling-Logo von 1970 hatte die visionäre Narration einer «Economics of the Coming Spaceship Earth», wie sie Kenneth Boulding I966 ausformuliert hatte, eine zeitgenössisch adaptierte visuelle Ikone gefunden. Eine solche «Spaceship-Economy» müsse sich, so Boulding, das rezyklierende Wirtschaften der Astronauten im begrenzten Raumschiff zum Vorbild nehmen und sei mithin ein Gegenbild zur bisherigen «Cowboy-Economy», welche auf eine Ausbeutung von Ressourcen ausgelegt sei. ${ }^{21}$ Recycling spielte auch in weiteren Werken der frühen Umweltbewegung eine Rolle und galt als Lösung für Umweltprobleme oder die Problematik begrenzter Ressourcen. ${ }^{22}$ Einige kritische Stimmen wiesen jedoch auch bereits auf die Beschränkungen und Unzulänglichkeiten des Recyclings hin, wofür erst in den I 99oer Jahren die Rede vom Downcycling aufkam. Der Wirtschaftsökonom Nicolas Georgescu-Roegen ging daher sogar so weit, dass er jedes Produzieren aus dem Blickwinkel dessen bewertete, was aus dem Gemachten zeitlich später werden würde - nämlich räumlich verteilte Abfälle: Ökonomische Wertschöpfung sei ein entropischer Prozess, der in der Natur vorkommende

Abb. 1 Der Uroboros auf dem Titelbild einer ersten amerikanischen Recycling-Studie, $197^{2}$

Abb. 2 Recycling-Logo von Gary Anderson, 1970

21 Vortrag auf dem «Resources for the Future Forum on Environmental Quality in a Growing Economy", vgl. Kenneth E. Boulding: Die Ökonomik des zukünftigen Raumschiffs Erde, in: Vereinigung für Ökologische Ökonomie - Beiträge und Berichte, Nr. 7: Beam us up, Boulding! 40 Jahre "Raumschiff Erde», 2006, 9-21.

22 Vgl. z. B. Donella Meadows u. a.: The limits to growth. A report for the Club of Rome's project on the predicament of mankind, New York 1972. 


\section{Recyclingfibel}
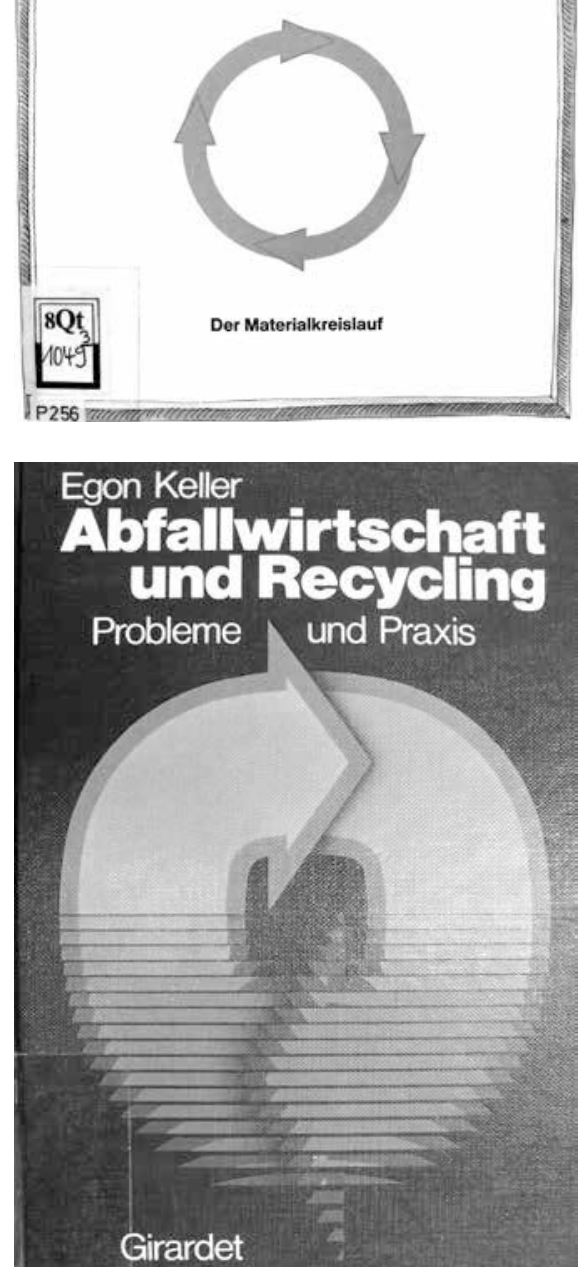

Abb. 3/4 Grüne KreislaufPiktogramme: Titelbilder von einschlägigen frühen RecyclingPublikationen, 1975 und 1977 kumulierte Rohstoffe abbaue und extrahiere, um daraus Produkte herzustellen, die schließlich als Abfall-Reste in Raum und Zeit dissipierten. ${ }^{23}$

In der Bildsymbolik der Handbücher und Ratgeber zur bundesrepublikanischen Abfallpolitik der i97oer Jahre dominieren grüne Recycling-Piktogramme, die aus heutiger Sicht an den Grünen Punkt des Dualen Systems Deutschland gemahnen, der seit 1990 die Konsument_innen tagtäglich zum getrennten Entsorgen von Verpackungen anhält. ${ }^{24}$ Die Broschüre Recyclingfibel. Der Materialkreislauf von 1975 zeigt auf dem Cover einen geschlossenen, aus vier grünen Pfeilen gebildeten Kreis (Abb. 3). Herausgeber war die Aktion saubere Landschaft e. V., eine I 970 von Vertreter_innen der Glas-, Verpackungs- und Getränkeindustrie sowie des Handels gegründete und auch von engagierten Bürger_innen mitgetragene Organisation, die gegen die zunehmende Verschmutzung von Stadtbild und Landschaft durch Konsummüll anging; finanzielle Unterstützung hatten das Bundesministerium des Inneren, an dem Umweltpolitik damals angesiedelt war, sowie das Umweltbundesamt geleistet. Laut Fibel bildete «im Sinne des Kreislaufdenkens» Müll «die Grundlage neuer Rohstoffe»; als Vorbild galt «die Natur». ${ }^{25}$ Die Fibel wollte den umweltbesorgten Bürger_innen Ratschläge für ihr Alltagsverhalten geben: Gartenabfälle könne man kompostieren, Verpackungsmaterial dem Lieferanten mitgeben, die - erst seit etwa einem Jahrzehnt üblich gewordene - Einkaufstüte mehrfach nutzen. Recycling wurde mithin als Aufgabe und Verantwortung der Bürger_innen dargestellt, die Müll reduzieren und trennen sollten. Wie Produktion und Abfallwirtschaft zum Gelingen von Recycling beitragen könnten, wurde demgegenüber kaum thematisiert.

In der BRD waren das Abfallgesetz von I972 sowie das Abfallwirtschaftsprogramm (AWP) von 1975 wichtige Etappen einer erstmals an ökologischen Kriterien ausgerichteten Abfallpolitik. Das Abfallgesetz sah den verpflichtenden Anschluss aller Haushalte an die Müllabfuhr vor, derweil noch Mitte der I96oer Jahre die Hälfte der Einwohner_innen ihren Müll anderweitig entsorgten. Veränderter Konsum und veränderte Müllzusammensetzungen hatten tradierte Entsorgungswege wie Müllgrube und Komposthaufen inzwischen auch auf dem Land an ihre Grenzen gebracht und angesichts eines steigenden Massenkonsums wurde das Wegwerfen von Resten zur Norm.

Die drei Kernsäulen des AWP waren das Recycling, eine umweltsensible Müllentsorgung sowie die Reduzierung von Abfällen - mithin Elemente, 
die bis heute die Debatten um den Abfall kennzeichnen und die im historischen Rückblick auf das AWP damals vor allem programmatischen Charakter hatten. Die bisher den Kommunen überlassene Abfallbeseitigung müsse, so das AWP, zu einer umfassenden «Abfallwirtschaft» werden, in der auch Produktion und Konsumtion umzugestalten seien, beispielsweise mittels gesteigerten Recyclat-Einsatzes in der Produktion, über recyclinggerechte Konstruktionen oder längere Produkthaltbarkeiten und -nutzungsphasen. ${ }^{26}$ Müllverbrennung solle die Energie im Müll effizienter nutzen; organische Reste könnten über das Kompostieren dem biologischen Kreislauf wieder zugeführt werden. Der Kreislauf beherrschte die Sprache des AWP und tauchte auch als Bild auf (Abb. 4): Die Publikation, über die Vertreter von Bund, Ländern, Kommunen und Industrie das AWP einer weiten Öffentlichkeit vorstellten, wählte für das Titelbild einen aus zwei ineinandergreifenden grünen Pfeilen geformten Kreis.

\section{Von Rucksäcken und Senken, Bäumen und Schmetterlingen: Abfall-Metaphern abseits des Kreislaufs}

Abfallpolitik wie auch der öffentliche Mülldiskurs drehten sich seit den I970er Jahren einseitig um den Hausmüll, auch wenn die Abfallberge der Produktion, die nun erstmals systematisch erfasst wurden, stetig wuchsen; sie sollten jene der Konsumtion quantitativ bald weit übertreffen. Die erfolgreiche Etablierung des Recycling-Logos, das zunehmende Umweltbewusstsein der Konsument_innen und ihre Mitmachbereitschaft beim Mülltrennen führten zu der paradoxen Situation, dass das Gesamtbild der Stoffumsätze, wie es Weyl für die Berliner Kanalisationsfrage zu erfassen gesucht hatte, kaum mehr in den Blick geriet. Dies änderte sich erst in den I 99oer Jahren. Recycling war inzwischen angesichts fehlender Entsorgungsmöglichkeiten - sogenannter <Senken〉, die all das Produzierte aufnehmen könnten - unumgänglich geworden, und das Abfallproblem hatte längst eine globale Dimension erreicht, wie zahlreiche Skandale um toxische oder illegale Müllexporte unmissverständlich anzeigten.

Es waren aber nicht allein diese Veränderungen, die einen Wandel im Denken zum Abfall auslösten; vielmehr spielte das Schaffen neuer Metaphern eine zentrale Rolle dabei, das Denken zu Rohstoffverbräuchen und Abfällen in neue Bahnen zu lenken. So kam der Downcycling-Begriff zu einer Zeit auf, als sich auch die Idee des <ökologischen Rucksackes〉 bzw. des <ökologischen Fußabdrucks> etablierte; dieses Konzept wurde unter anderem von Friedrich Schmidt-Bleek am Wuppertal Institut für Klima, Umwelt und Energie im Kontext der Berechnungen zum Material-Input pro Serviceeinheit (MIPS) verwendet. ${ }^{27}$ Gemeint ist damit die Menge der Stoffe, die beim Produzieren, Nutzen und Entsorgen eines Produkts verwendet wird - und mithin als Rest anfällt. Die Initiatoren des cradle-to-cradle-Ansatzes, William McDonough
23 Nicolas Georgescu-Roegen: Was geschieht mit der Materie im Wirtschaftsprozeß? Recycling: Lösung der Umweltkrise?, in: Brennpunkte, Nr. 2, 1974, 17-28. 24 Dessen Urheber, Lars Oehlschlaeger, beruft sich auf das Yin-Yang-Symbol als Vorbild, vgl. Melanie Amann: Volk der Trenner und Sammler, in: Frankfurter Allgemeine Zeitung, 31.10.2010.

25 Aktion saubere Landschaft (Hg.): Recyclingfibel. Der Materialkreislauf, Bonn 1975, 8.

26 Vgl. zum AWP Egon Keller (Hg.): Abfallwirtschaft und Recycling. Probleme und Praxis, Essen 1977.

27 Siehe ebenso Mathis Wackernagel, William E. Rees: Unser ökologischer Fußabdruck. Wie der Mensch Einfluß auf die Umwelt nimmt, Basel u. a. 1997. 


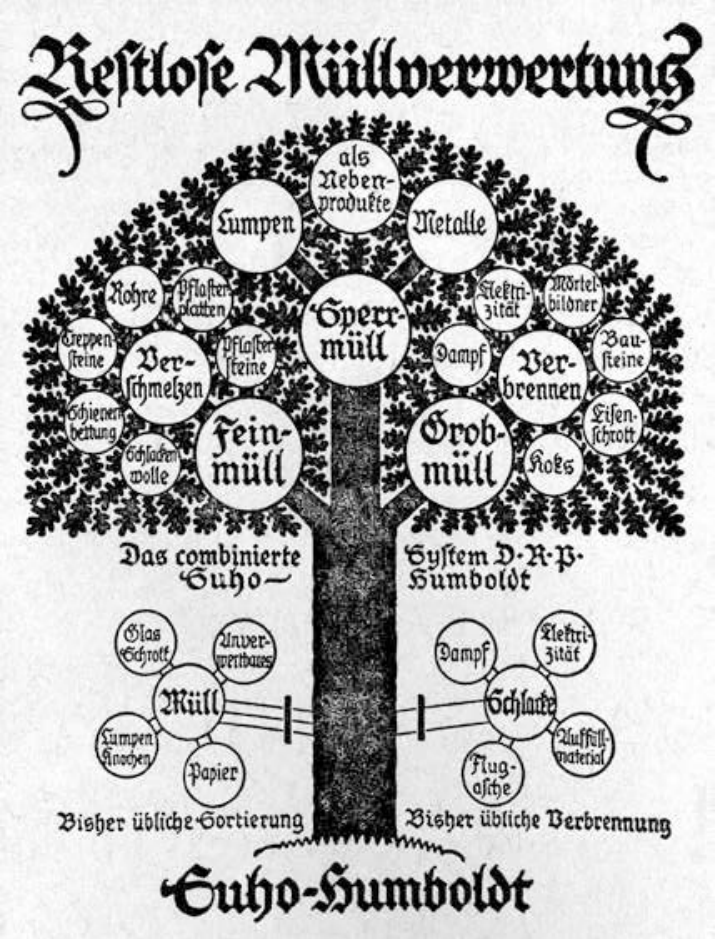

und Michael Braungart, rechneten wenig später sogar vor, dass ein Produkt im Durchschnitt nur fünf Prozent der dafür extrahierten und aufgebrachten Materialien enthalte: cradle-to-grave statt cradle-to-cradle beherrsche mithin das bisherige Wirtschaften. ${ }^{28}$ Inzwischen findet sich der Ansatz, dem «Lebenszyklus» eines Produkts und seinen $<$ Rucksäcken $>$ zu folgen, auch in Technik- und Medienstudien wieder. So untersucht Kyle Devine den Konsum von Musikaufnahmen, der zunächst auf Schellack, dann auf Kunststoff und schließlich auf digitalen Techniken basierte, auf die damit einhergehenden Ressourcen- und Energieverbräuche und Abfallmengen; ${ }^{29}$ Josh Lepawsky erinnert daran, dass zu den discardscapes des Elektronikschrotts nicht nur die weggeworfenen Mediengeräte gehören, sondern auch die Abraumhalden der Erzschürfung, Überreste der Produktion und die Verbräuche und Emissionen, die mit jedem digitalen Medienakt einhergehen..$^{\mathbf{3 0}}$

Es ist der Kirschbaum, mit dem Braungart die cradle-to-cradle-Idee $\mathrm{zu}$ visualisieren suchte: ${ }^{31}$

Abb. 5 Der Baum als Bild für das Müllverwertungsverfahren «Suho-Humboldt» der Musag auf dem Titelblatt der Zeitschrift Die Städtereinigung, $192 \mathrm{I}$
$28 \mathrm{Vgl}$. William McDonough, Michael Braungart: Cradle to Cradle. Remaking the Way We Make Things, New York 2002, 28.

29 Kyle Devine: Decomposed. The Political Ecology of Music, Cambridge u. a. 2019.

30 Josh Lepawsky: Reassembling Rubbish. Worlding Electronic Waste, Cambridge u. a. 2018.

31 McDonough u. a.: Cradle to Cradle, $72 \mathrm{f}$.
Produzieren und Konsumieren könnten so üppig, verschwenderisch und gleichzeitig nachhaltig sein wie ein Kirschbaum, der überzählige Blüten produziert, von denen nur wenige zu Kirschen werden, deren Reste aber im ewigen Nährstoffkreislauf der Natur niemals verloren gehen. Dazu müssten die technischen Kreisläufe nur ebenso geschlossen funktionieren wie die natürlichen. Diese Parallel-Setzung übersieht jedoch, dass ein Baum über Jahrzehnte, gar Jahrhunderte wächst und die Zyklen der Natur sowie ihre biochemischen Stofftransformationen ihren je eigenen Zeitverläufen folgen. Das Uroboros-Prinzip eines ewigen Werdens und Vergehens der Natur, die keine Abfälle kennt, und seine organisch-fossile Zeit lassen sich nicht mit der Kultur und Wirtschaft einer produzierenden und konsumierenden Gesellschaft, in der unweigerlich Reste entstehen, und ihren kulturellen und technisch-ökonomischen Zeittaktungen parallelisieren.

In seiner bildlichen Verwendung hat der Baum jedoch eine andere epistemische Tradition: Der verzweigte Baum visualisiert Abstammungsverhältnisse und zeitlich geordnete Abfolgen und Verzweigungen, durch welche das Vorherige das Folgende erzeugt, und damit verschiedenste Enden eines gemeinsamen Stamms etwa von Lebewesen, Familien oder Stoffen und ihren Derivaten. Anfang der I92oer Jahre verbildlichte der Müllentsorger Musag sein Verfahren zur Müllaufbereitung mit einer solchen Baum-Figur (Abb. 5), die seitdem aber nicht mehr im Mülldiskurs verwendet worden ist. Versprochen wurde eine «restlose 
Müllverwertung», bei der Müll «nicht mehr ein störender, wertloser Abfall, dessen Vernichtung große Kosten verursacht» sei, sondern Quell neuer Werte. ${ }^{32}$ Der Baum wurzelte im Suho-Humboldt-Verfahren, trug die Blätter der Eiche und verzweigte sich in drei große Äste - die Haupt-Müllfraktionen von Fein-, Sperr- und Grobmüll -, aus denen dann weitere Verästelungen mit unterschiedlichen Wertstoffen als $<$ Früchten $>$ hervorgingen. Der Grobmüll (rechter Ast) wurde

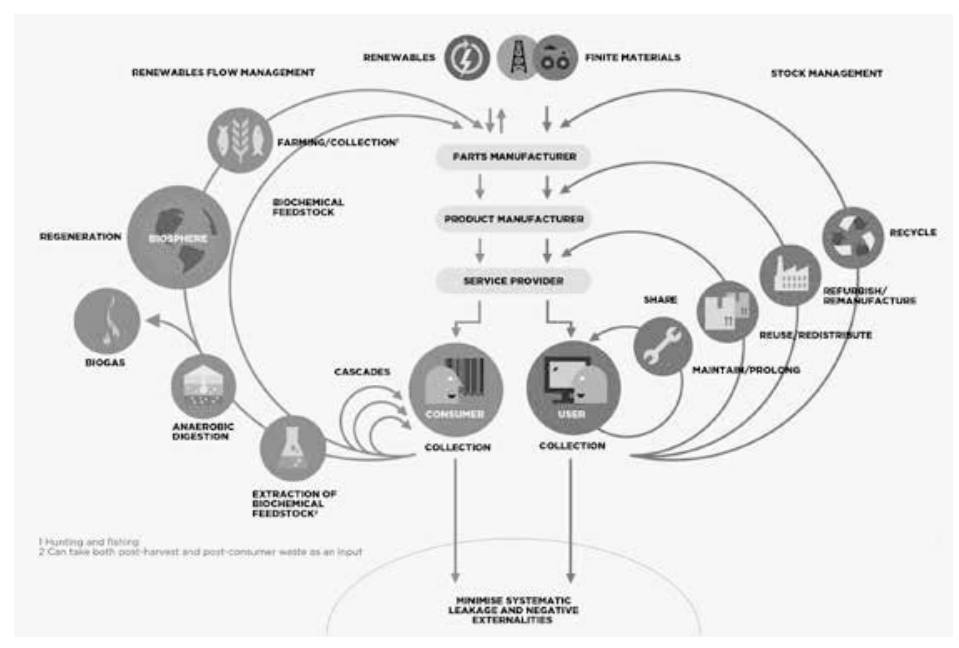
verbrannt, wobei in diesem Prozess

Dampf, Elektrizität und Koks entstanden; möglicher Eisenschrott wurde aus der Schlacke aussortiert, ehe diese zu Mörtel und Bausteinen weiterverarbeitet wurde. Der Feinmüll (linker Ast) wurde mit Zusätzen niedergeschmolzen, um diverse Baumaterialien zu gewinnen. Beim Sperrmüll (mittlerer Ast) wurden vornehmlich Lumpen und Metalle wiedergewonnen. Die bisherige Müllsortierung bzw. Müllverbrennung wurde demgegenüber als inferior gedeutet: Sie war im unteren Baumbereich als beidseitiger, frühzeitiger und verkümmerter Fehlwuchs angedeutet.

Auch wenn die Vision der circular economy auf der Kreislauf-Metapher gründet, so hat die Ellen-MacArthur-Stiftung für deren Propagierung ein neues Bild entwickelt: das sogenannte Schmetterlings-Diagramm (Abb. 6). Es ordnet die Akteure von Herstellung, Dienstleistung und Konsum als Hauptlinie oder Stamm zwischen den Ressourcen (oben) und Abfallsenken (unten) an; links und rechts davon gehen wie zwei Schmetterlingsflügel die sich dann wiederum verästelnden Kreisläufe der «biologischen» bzw. «technischen» Nährstoffe ab. Damit ist das Schmetterlings-Diagramm letztlich Hybrid aus Flussdiagramm, Kreislauf- und Baum-Figur, und derweil die einzelnen Transformationsprozesse - etwa von Waren durch Praktiken wie Reparieren, Sharing oder Recycling - differenziert werden, verbleiben die In- und Output-Ströme, die in das System hinein- bzw. aus ihm herausführen, im Vagen: Pfeile unten deuten das Entweichen von minimalen Sickerverlusten an, die einer Senke bedürfen. Die Hoffnung des Diagramms liegt in der Minimierung dieser Ausscheidungen, um das Problem der fehlenden Senken zu lösen - eine Hoffnung, die in weiter Ferne liegt, wie es ein Blick auf die Gesamtbilanz der aktuellen Stoffströme unmittelbar nahelegt: Denn nicht einmal zehn Prozent aller extrahierten und genutzten Stoffe werden derzeit rezkyliert. ${ }^{33}$ «The world is now only $8.6 \%$ circular», berichtet so auch die Initiative Circle Economy in ihrem Bericht von 2020.34 Dem Hoffen auf

Abb. 6 Schmetterlingsdiagramm der angedachten Kreislaufwirtschaft. Quelle: Ellen MacArthur Foundation
32 Hinter der Musag verbarg sich die Gesellschaft für den Bau von Müll- und Schlackenverwertungsanlagen, ein Zusammenschluss der Kölner Maschinenbau-Anstalt Humboldt und des Schmelz- und Hüttenwerks Oberschöneweide. Für das Folgende vgl. Anzeige in: Die Städtereinigung, Bd. 15, 1.8.1921, Titelblatt; Restlose Müllverwertung nach dem System Suho des Schmelzund Hüttenwerks Oberschöneweide - Humboldt Köln/Kalk, in: Die Städtereinigung, Bd. 14, 1921, 106-110.

33 Die Zahl wird auch in einem Flussdiagramm im eingangs zitierten National-Geographic-Bericht genannt, vgl. Kunzig: Raus aus dem Müll, 46 .

34 www.circularity-gap.world (5.5.2020). 
den Kreislauf scheint dies aber keinen Abbruch zu tun: Die Differenz - also der Verlust in der materialökonomischen Gesamtbilanz - wird schlichtweg als circularity gap angesprochen.

\section{Fazit: Der Kreislauf als ewiges Heilsversprechen}

Der circularity gap schließt sich jedoch nicht von selbst und er ist zudem nicht historisch neu, wie es eine weitere aktuelle Metapher zeigt: diejenige der «Technofossilien». Unter diesem in der Anthropozän-Debatte situierten Stichwort untersuchen Geolog_innen derzeit die Gesamtmenge der vom Menschen gemachten materiell-technischen Hinterlassenschaften. Beziffert wird die Menge dieser Technofossilien auf rund 30 Billionen Tonnen Material bei mehr als I 70.000 Arten an «synthetisch-mineralischen Substanzen». ${ }^{35} \mathrm{Da}-$ bei stieg diese Menge ab den r 95oer Jahren expansiv an, ein Prozess, den Umwelthistoriker_innen inzwischen als «great acceleration» bezeichnen. ${ }^{36}$ Insbesondere Bilder von Ausscheidungen der Gegenwart kursieren auch zahlreich in den Medien oder der Kunstwelt. So hat Edward Burtynsky in seiner Technofossils-Serie diverse Abfallberge von alten Telefon-Wählscheiben, $e$-waste, Plastik etc. fotografiert. ${ }^{37}$ Diese Bilder gemahnen daran, dass trotz einer angeblich bereits etablierten $<$ Kreislaufwirtschaft $〉$ große Mengen problematischer Reste als nutzlos aus dem Wirtschaftssystem ausgestoßen werden. Sie erinnern aber auch daran, dass im Gesamtsystem der Erde nichts verloren geht: Zahlreiche Hinterlassenschaften insbesondere der jüngeren Vergangenheit bereiten uns und den kommenden Generationen mehr und mehr Probleme, seien es industrielle <Altlasten $>$ und <Rückstände $>$ in den <Umweltmedien $>$ Boden, Luft oder Wasser oder die seit der Industrialisierung steigenden CO 2-Ausstöße oder der in $<$ Endlager $>$ zu verbringende atomare Müll.

Die Bilder von Abfallsenken und -bergen oder eines <ökologischen Fußabdrucks > sind inzwischen im allgemeinen Umweltbewusstsein verankert. Dennoch treibt die extrem reduktionistische Kreislauf-Metapher - meist beschränkt sich das Bildvokabular auf Kreis, Pfeil und die Farbe Grün - weiterhin das aktuelle Diskutieren, Handeln und Hoffen im Abfallbereich an. Wie gezeigt wurde, war sie als Denkfigur schon über Jahrhunderte hinweg präsent: Als Sprachbild und mit Aufkommen der Recycling-Politik vermehrt

35 Erle C. Ellis: Anthropocene. A Very Short Introduction, Oxford 2018, 148. 36 Peter Engelke, John McNeill: The Great Acceleration. An Environmental History of the Anthropocene since 1945, Cambridge 2014.

37 Edward Burtynsky, Jennifer Baichwal, Nicholas de Pencier: Anthropocene, Göttingen 2018. 38 Siehe z. B. das 2018 gegründete Fraunhofer-Cluster Circular Plastics Economy, ccpe.fraunhofer.de (3.5.2020) in visueller Form diente sie einerseits dazu, Reste-Ökonomien konzeptionell zu fassen - den Lumpenhandel ebenso wie die Flüsse von Fäkalien und die darin enthaltenen Nährstoffe oder das heutige Plastik-Recycling. ${ }^{38}$ Andererseits wurden mit Hilfe der Kreislauf-Metapher zugleich auch Mängel im bestehenden Abfallumgang diagnostiziert und mehr Recycling von Resten eingefordert. Allerdings ist sie weder epistemisch noch repräsentational sinnvoll. Denn sie kann die Verhältnisse des Recyclings und darin inkludierte Prozesse wie Müll-, Trenn- und Transportarbeit und unvermeidliche Verluste nicht adäquat erfassen. 
Ähnlich wie in der Debatte zur Frage der Ableitung von Fäkalien aus den Städten um I900 übernimmt die Kreislauf-Metapher jedoch auch aktuell eine politisch-ideelle Funktion: Sie dient als Heilsversprechen, dass die Massenkonsumgesellschaft ihr Abfallproblem und die Folgen der von ihr massiv veränderten Stoffströme der Erde auf technisch-ökonomischem Wege wird lösen können; als Beleg dient die Unhintergehbarkeit des Kreislauf-Prinzips der Natur. Wie es der kurze historische Abriss nahelegt, hat dieses Heilsversprechen die Geschichte von Resten begleitet und es kehrt offenbar immer dann zyklisch wieder, wenn sich eine Gesellschaft in Übergangsphasen befindet, während derer sich der gesellschaftliche Stoffwechsel fundamental ändert - in diesem Sinne scheint es sich also um ein <ewiges> Heilsversprechen zu handeln. 